\title{
Endothelial Function and Cardiovascular Autonomic Activity in Neurally Mediated Syncope
}

\author{
Kyoung-Ha Park Sang Jin Han Hyun-Sook Kim Sang Ho Jo Sung-Ai Kim \\ Woo Jung Park \\ Cardiovascular Division, Department of Internal Medicine, Hallym University Medical Center, Anyang, Republic of Korea
}

\author{
Key Words \\ Neurally mediated syncope Endothelial function . \\ Parasympathetic activity
}

\begin{abstract}
Objectives: The aim of this study was to investigate endothelial function and cardiovascular autonomic activity in patients with neurally mediated syncope (NMS). Methods: Patients with a typical history of NMS were divided according to the result of a head-up tilt (HUT) test. There were 25 patients each in the HUT-positive (HUT+), HUT-negative (HUT-) and control groups. Flow-mediated dilation (FMD) and 24-hour ambulatory electrocardiography (AECG) were performed before the HUT tests. Results: The HUT+ group had a significantly higher FMD than that of the HUT- group and the control group $(8.8 \pm 3.3$ vs. $6.4 \pm 2.9 \%, p=0.006$, and $8.8 \pm 3.3$ vs. $5.7 \pm 2.2 \%, p=0.001$, respectively). On a 24 -hour AECG, the parasympathetic indexes of time domain, such as rMSSD and the pNN50, were significantly higher in the HUT+ group than in the HUT- group (39.0 \pm 9.6 vs. $31.6 \pm 9.6 \mathrm{~ms}$, $\mathrm{p}=0.016$, and $16.5 \pm 8.1$ vs. $10.2 \pm 7.2 \%, \mathrm{p}=0.002$, respectively) and the control group (39.0 \pm 9.6 vs. $28.9 \pm 9.6 \%, p=$ 0.001 and $16.5 \pm 8.1$ vs. $8.7 \pm 6.7 \%, p=0.001$, respectively). High-frequency spectra (parasympathetic activity) of the fre-
\end{abstract}

quency domain showed similar results. Conclusions: Not only parasympathetic activity, but also endothelial function may affect the results of HUT tests in patients with NMS.

(c) 2016 S. Karger AG, Basel

\section{Introduction}

The pathophysiologic mechanism of neurally mediated syncope (NMS) involves changes in the autonomic nervous system and results in marked hypotension with reflex bradycardia $[1,2]$. The head-up tilt (HUT) test is a useful method that is able to identify patients affected by NMS. However, previous studies reported that the results of HUT tests are not always reproducible in individual patients with NMS $[3,4]$. Heart rate variability (HRV) derived from an ambulatory electrocardiogram (AECG) provides useful information about cardiac autonomic activity, which is associated with the pathogenesis of NMS $[5,6]$. In patients with NMS, a few studies have reported changes in peripheral vascular tone, but the results were controversial $[7,8]$. One possible reason is that patients with NMS could have variations in endothelial function and cardiac autonomic activity, which may affect peripheral vasomotion. However, the cause of individual sus-

\section{KARGER}

E-Mail karger@karger.com

www.karger.com/crd
(C) 2016 S. Karger AG, Basel

$0008-6312 / 16 / 1342-0065 \$ 39.50 / 0$
Dr. Kyoung-Ha Park

Cardiovascular Division, Department of Internal Medicine

Hallym University Medical Center, 896 Pyeongchon-dong, Dongan-gu

Anyang-si, Gyeonggi-do (Republic of Korea)

E-Mail pkhmd@ naver.com 
ceptibility to NMS has not been well investigated. The aim of the present study was to evaluate the activity of peripheral endothelial function and its relationship with cardiovascular autonomic activity, using AECG indices in patients with NMS.

\section{Methods}

\section{Study Population}

Patients with at least one episode of suspected NMS within a 2 -month period prior to visiting the hospital were evaluated. NMS was suspected when the patient had the following typical symptoms: nausea, light headaches, a feeling of warmth, pallor and cold sweat before the abrupt loss of consciousness, the cause of which could not be determined despite a careful review of the medical history and a comprehensive close examination, including a neurological examination, carotid Doppler ultrasound, 12-lead ECG, 24-hour AECG, echocardiography and exercise treadmill test. Patients over 40 years of age or who had structural cardiovascular disease, hypertension, diabetes, ischemic heart disease, Wolff-Parkinson-White syndrome, bundle branch block, premature beats $>2 \%$ of total beats, absence of a sinus rhythm or serious arrhythmia were excluded from the study. To compare endothelial function and HRV according to the HUT results, we enrolled three groups of patients: (1) those with NMS and a positive HUT test (HUT+ group), (2) those with strongly suspected NMS on medical history and clinical examination but with a negative HUT test (HUTgroup) and (3) healthy age- and sex-matched controls (control group).

\section{$H R V$ Indices}

Autonomic cardiovascular activity was evaluated in all subjects with 24-hour AECG at least $24 \mathrm{~h}$ prior to HUT. AECG monitoring was performed using a three-channel (leads V5, V1 and aVF) digital recorder (SEER Light; GE, Fairfield, Conn., USA). All of the data were subsequently analyzed to measure HRV using a commercially available program (MARS Holter software; GE). The time domain analysis of HRV included the mean of all normal R-R intervals (mean NN), the standard deviation (SD) of the NNs (SDNN), and the SD of the 5-min mean values of the NNs (SDANN). As indexes of sympathovagal balance, in particular with parasympathetic tone, the rMSSD (root mean square successive difference of NNs) and the pNN50 (proportion of successive $\mathrm{NN}$ differences of $>50 \mathrm{~ms}$ for each 5-min interval) were analyzed [9]. As frequency domain parameters expressing the overall HRV, parasympathetic (high-frequency spectra, $0.15-0.40 \mathrm{~Hz}$ ) and sympathetic (low-frequency spectra, $0.04-0.15 \mathrm{~Hz}$ ) activity was measured [9].

\section{Endothelial Function and Peripheral Vasomotion}

The studies were performed at least $24 \mathrm{~h}$ before HUT testing. The patients were instructed not to exercise or to ingest substances that might affect flow-mediated dilation (FMD), such as caffeine, food or tobacco for at least $12 \mathrm{~h}$ before the study. They were instructed to avoid vitamin supplements for at least $72 \mathrm{~h}$ before the study. The patients were evaluated in the morning in a quiet airconditioned room $\left(23-25^{\circ} \mathrm{C}\right)$. An experienced vascular sonogra- pher who was blinded to the patients' information performed an ultrasound examination using a high-resolution B-mode scan, which was obtained with a $10.0-\mathrm{MHz}$ linear-array transducer $(\mathrm{Hi}-$ tachi Aloka Medical, Tokyo, Japan). The transducer was held throughout the scan at the same point by a dedicated stereotactic probe-holding device (MP-PH0001, Hitachi Aloka Medical). During the study, specified computer-assisted analysis software was used with an automated continuous-edge tracking system for the measurement of arterial diameter (Prosound- $\alpha 7$, Hitachi Aloka Medical). Briefly, the right brachial artery (RBA) was scanned over a longitudinal section $2-5 \mathrm{~cm}$ above the antecubital fossa. To obtain good-quality images, the frames were synchronized on the Rwave of the ECG. After a longitudinal image of the artery was acquired, a blood pressure cuff that had been placed around the forearm just below the elbow was inflated to $50 \mathrm{~mm} \mathrm{Hg}$ above systolic pressure for $5 \mathrm{~min}$. After cuff deflation, the longitudinal image of the artery was recorded continuously until 5 min after cuff deflation. After reactive hyperemia, changes in brachial artery diameter were immediately expressed as the maximal percent change relative to the vessel diameter before cuff inflation, and FMD (endothelium-dependent vasodilation) was calculated. After $15 \mathrm{~min}$ of FMD measurement and the restoring of baseline resting images, a sublingual nitroglycerin $(0.6 \mathrm{mg})$ was administered and images of the artery were recorded for 5 min for nitrate-mediated dilation (NMD; endothelium-independent vasodilation). The response of the artery diameter was automatically calculated as the percent change in peak vessel diameter from the baseline value. The response of the arterial diameter was automatically calculated as the percent change in peak vessel diameter from the baseline value. The percent values of FMD and NMD (peak diameter - baseline diameter/baseline diameter $\times 100$ ) were used for analysis. In our laboratory, the Lin's concordance correlation coefficient values for the interobserver and intraobserver agreement of FMD (0.867 and 0.835 , respectively) and NMD (0.845 and 0.802 , respectively) were of an acceptable level.

\section{HUT Test}

After overnight fasting, the subjects underwent HUT in the morning in a quiet air-conditioned room $\left(23-25^{\circ} \mathrm{C}\right)$. An electronically controlled tilt table with a foot board for weight support was used. The HUT protocol consisted of at least $15 \mathrm{~min}$ in the supine position under stable conditions, followed by a 60-degree passive tilt for $45 \mathrm{~min}$. Electrocardiography was monitored continuously and blood pressure was measured at 1-min intervals using an automatic sphygmomanometer (BP 203, Nihon Colin Inc., Tokyo, Japan). If the patient presented a negative response to the baseline HUT, a 20-min period of isoproterenol provocation testing was performed. Based on the previous report that the positive response to low-dose isoproterenol was higher than nitroglycerin in younger patients compared with older patients [10-12], we used the lowdose isoproterenol provocation test because we excluded patients over 40 years of age as well as high-risk patients. For the isoproterenol provocation test, an intravenous isoproterenol infusion was started at a rate of $1-3 \mu \mathrm{g} / \mathrm{min}$ to obtain a $25 \%$ increase in heart rate compared to control supine values. HUT was stopped at the induction of the syncope, presyncope or the completion of $45 \mathrm{~min}$ of passive tilt and 20 min of provocation test. A positive HUT occurred when the patient's syncopal or presyncopal symptoms were reproduced and accompanied by hypotension, bradycardia (relative or otherwise) or both. 
Table 1. Baseline clinical characteristics

\begin{tabular}{lcccc}
\hline & $\begin{array}{l}\text { HUT+group } \\
(\mathrm{n}=25)\end{array}$ & $\begin{array}{l}\text { HUT-group } \\
(\mathrm{n}=25)\end{array}$ & $\begin{array}{l}\text { Control group } \\
(\mathrm{n}=25)\end{array}$ & p value \\
\hline Age, years & $28 \pm 5$ & $29 \pm 6$ & $29 \pm 6$ & 0.742 \\
Male gender & $5(20)$ & $8(32)$ & $6(24)$ & 0.611 \\
Current smoking & $3(12)$ & $2(8)$ & $3(12)$ & 0.869 \\
Dyslipidemia & $1(4)$ & $0(0)$ & $0(0)$ & 0.807 \\
BMI & $25.2 \pm 2.4$ & $24.8 \pm 2.5$ & $25.1 \pm 2.4$ & 0.851 \\
Systolic blood pressure, mm Hg & $117 \pm 12$ & $119 \pm 10$ & $115 \pm 12$ & 0.443 \\
Diastolic blood pressure, mm Hg & $67 \pm 6$ & $69 \pm 6$ & $68 \pm 8$ & 0.782 \\
Heart rate, beats/min & $65 \pm 8$ & $64 \pm 10$ & $64 \pm 8$ & 0.722 \\
Hemoglobin, g/dl & $13.7 \pm 1.5$ & $14.2 \pm 1.3$ & $14.5 \pm 1.7$ & 0.259 \\
Fasting blood sugar, mg/dl & $105 \pm 11$ & $100 \pm 12$ & $108 \pm 15$ & 0.115 \\
Total cholesterol, mg/dl & $183 \pm 29$ & $179 \pm 29$ & $189 \pm 24$ & 0.433 \\
Creatinine, mg/dl & $0.7 \pm 0.3$ & $0.7 \pm 0.2$ & $0.7 \pm 0.2$ & 0.992 \\
\hline
\end{tabular}

Data are presented as $\mathrm{n}(\%)$ or mean $\pm \mathrm{SD}$.

\section{Statistical Analysis}

On the basis of the results of our pilot study, we assumed that the difference of FMD would be $2.0 \%$ higher in the HUT+ group compared to the HUT- group. We used a two-sided level of 0.05 and SD of $2.1 \%$, which was based on our pilot study showing that the difference in the mean between the HUT+ group and the HUT - group was $2.9 \pm 2.1 \%$. We estimated that 50 patients ( 25 per group) were needed to demonstrate the FMD differentiate between the groups with a statistical power of $80 \%$. The data are expressed as the mean \pm SD. The obtained variables were tested for normal distribution with the Kolmogorov-Smirnov test. The values obtained in each group and the differences between measurements were analyzed by the independent Student $t$ test or the Mann-Whitney U test as appropriate. Analysis of variance was used to assess mean differences among the three groups. Multivariate logistic regression analysis was performed to investigate interactions between FMD and relevant autonomic indices of HRV for positive HUT. Since gender could be an important variable in the analysis, we also included female gender in the multivariate analysis [13-15]. Differences were considered significant at values of $\mathrm{p}<0.05$. All statistical procedures were performed using SPSS 19.0 (SPSS Inc., Chicago, Ill., USA). The protocol was approved by the Ethics Committee of Hallym University Medical Center and all patients and healthy control subjects gave informed written consent.

\section{Results}

Between May 2013 and April 2014, 107 consecutive patients with suspected NMS were assessed for this study. Of these, 57 patients were excluded because they were aged $>40$ years $(n=24)$, had structural heart disease $(n=$ $3)$, diabetes $(n=8)$, hypertension $(n=13)$, bundle branch block $(n=2)$, premature beats $>2 \%$ of total beats $(n=5)$ or absence of a sinus rhythm $(n=2)$, leaving 50 patients enrolled in the study. According to the results of HUT testing, 25 patients were assigned to the HUT + group and 25 patients were assigned to the HUT-group. According to the Vasovagal Syncope International Study (VASIS) criteria, there were 16 patients of mixed type (VISIS-1), 3 patients of cardioinhibitory type (VASIS-2A in 1 and VASIS-2B in 2), and 6 patients of pure vasodepression type (VASIS-3) in the HUT+ group [16]. Twenty-five subjects were also enrolled in the healthy control group. The subjects in the HUT+ group, the HUT- group and the control group were comparable regarding age, gender, cardiac risk factors, BMI, systolic and diastolic BP, lipid profile and resting heart rate (table 1). In the HUT+ group, 11 patients had syncope induced by the drug-free phase of HUT. The remaining 14 patients underwent the isoproterenol provocation test.

The results of the FMD and NMD studies are shown in table 2. The comparison of the RBA vessel diameters among the groups was equivalent. The mean value of FMD in the HUT+ group was $8.8 \pm 3.3 \%$. This value was significantly greater than that of the HUT-group ( $8.8 \pm$ 3.3 vs. $6.4 \pm 2.9 \%, p=0.006)$ and that of the control group ( $8.8 \pm 3.3$ vs. $5.7 \pm 2.2 \%, p=0.001$; fig. 1a). In terms of NMD, the mean value of NMD in the HUT+ group was $25.2 \pm 4.6 \%$. This value was not different from that of the HUT- group ( $25.2 \pm 4.6$ vs. $24.4 \pm 6.4 \%, \mathrm{p}=0.596)$ and that of the control group ( $25.2 \pm 4.6$ vs. $26.2 \pm 5.9 \%, \mathrm{p}=$ 0.541; fig. 1b). 
Fig. 1. Comparison of FMD and NMD of the RBA. a The mean value of FMD in the $\mathrm{HUT}+$ group was significantly greater than that of the HUT- group $(* \mathrm{p}=0.006)$ and the control group $\left({ }^{* *} \mathrm{p}=0.001\right)$. $\mathbf{b}$ In terms of NMD, there was no difference among the groups. Error bars depict the 95\% confidence intervals.

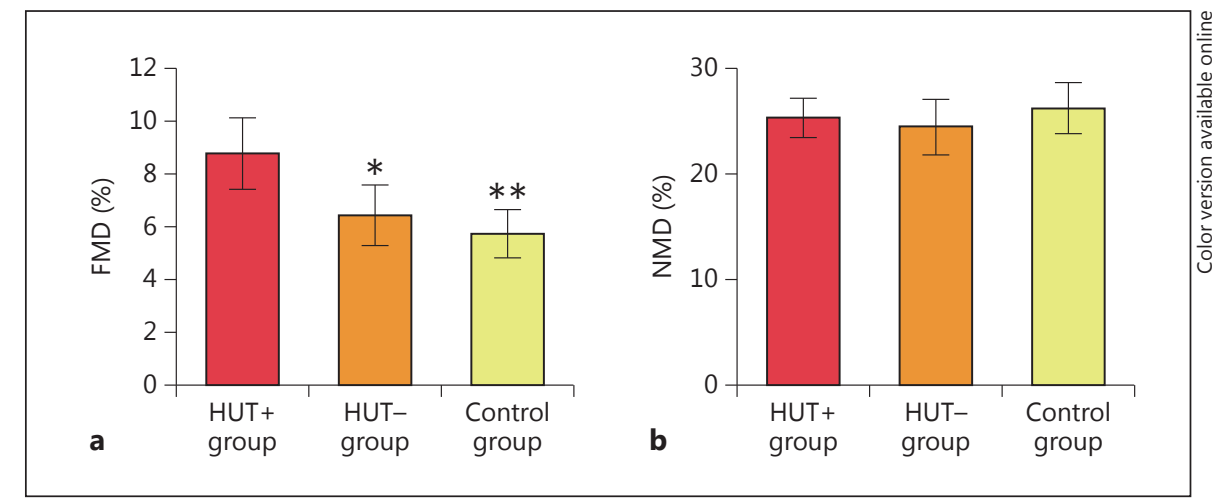

Table 2. Results of endothelial and peripheral vasomotion studies in the RBA

\begin{tabular}{|c|c|c|c|c|}
\hline & $\begin{array}{l}\text { HUT }+ \text { group } \\
(\mathrm{n}=25)\end{array}$ & $\begin{array}{l}\text { HUT-group } \\
(\mathrm{n}=25)\end{array}$ & $\begin{array}{l}\text { Control group } \\
(\mathrm{n}=25)\end{array}$ & $\mathrm{p}$ value \\
\hline Baseline diameter of the RBA, mm & $3.44 \pm 0.45$ & $3.54 \pm 0.63$ & $3.48 \pm 0.54$ & 0.773 \\
\hline \multicolumn{5}{|l|}{ Endothelium-dependent vasodilation } \\
\hline Peak diameter of the RBA, $\mathrm{mm}$ & $3.74 \pm 0.47$ & $3.77 \pm 0.64$ & $3.69 \pm 0.58$ & 0.870 \\
\hline FMD, $\%$ & $8.8 \pm 3.3^{\mathrm{a}, \mathrm{b}}$ & $6.4 \pm 2.9$ & $5.7 \pm 2.2$ & 0.001 \\
\hline \multicolumn{5}{|l|}{ Endothelium-independent vasodilation } \\
\hline Peak diameter of the RBA, mm & $4.30 \pm 0.53$ & $4.39 \pm 0.66$ & $4.40 \pm 0.71$ & 0.830 \\
\hline NMD, \% & $25.2 \pm 4.6$ & $24.4 \pm 6.4$ & $26.2 \pm 5.9$ & 0.551 \\
\hline
\end{tabular}

Table 3. Comparison of HRV among the HUT+, HUT- and control groups

\begin{tabular}{lcccc}
\hline & $\begin{array}{l}\text { HUT+group } \\
(\mathrm{n}=25)\end{array}$ & $\begin{array}{l}\text { HUT-group } \\
(\mathrm{n}=25)\end{array}$ & $\begin{array}{l}\text { Control group } \\
(\mathrm{n}=25)\end{array}$ & p value \\
\hline Mean NN, ms & $863.6 \pm 94.5$ & $856.5 \pm 94.5$ & $822.8 \pm 97.5$ & 0.277 \\
SDNN, ms & $150.1 \pm 34.7$ & $138.2 \pm 26.3$ & $135.3 \pm 26.7$ & 0.177 \\
SDANN, ms & $135.3 \pm 37.3$ & $123.2 \pm 28.0$ & $121.9 \pm 25.6$ & 0.230 \\
rMSSD, ms & $39.0 \pm 9.6^{\mathrm{a}, \mathrm{b}}$ & $31.6 \pm 9.6$ & $28.9 \pm 9.6$ & 0.001 \\
pMN50, \% & $16.5 \pm 8.1^{\mathrm{c}, \mathrm{d}}$ & $10.2 \pm 7.2$ & $8.7 \pm 6.7$ & 0.001 \\
High-frequency domain, ms & $16.4 \pm 4.7^{\mathrm{e}, \mathrm{f}}$ & $13.2 \pm 5.0$ & $12.5 \pm 4.9$ & 0.012 \\
Low-frequency domain, ms & $22.0 \pm 5.3^{\mathrm{g}}$ & $18.7 \pm 7.3$ & $18.5 \pm 4.9$ & 0.069 \\
\hline
\end{tabular}

${ }^{\mathrm{a}} \mathrm{p}=0.016$ vs. HUT-; ${ }^{\mathrm{b}} \mathrm{p}=0.001$ vs. control; ${ }^{\mathrm{c}} \mathrm{p}=0.002$ vs. HUT-; ${ }^{\mathrm{d}} \mathrm{p}=0.001$ vs. control; ${ }^{\mathrm{e}} \mathrm{p}=0.024$ vs. HUT-; ${ }^{\mathrm{f}} \mathrm{p}=0.005$ vs. control; ${ }^{\mathrm{g}} \mathrm{p}=0.019$ vs. control.

On 24-hour AECG, there were no significant differences among the groups on three time-domain HRV indices: mean NN, SDNN and SDANN (table 3). In the other time domains of the HRV indices, the HUT+ group presented with a significantly higher $\mathrm{MSSD}$ and pMN50 than that of the HUT-group ( $39.0 \pm 9.6$ vs. $31.6 \pm 9.6 \mathrm{~ms}$, $\mathrm{p}=0.016$, and $16.5 \pm 8.1$ vs. $10.2 \pm 7.2 \%, \mathrm{p}=0.002$, respec- tively) and the control group (39.0 \pm 9.6 vs. $28.9 \pm 9.6 \%$, $\mathrm{p}=0.001$, and $16.5 \pm 8.1$ vs. $8.7 \pm 6.7 \%, \mathrm{p}=0.001$, respectively). In terms of the frequency domain, the HUT+ group showed higher parameters in the high-frequency domain compared with the HUT-group and the control group $(16.4 \pm 4.7$ vs. $13.2 \pm 5.0 \mathrm{~ms}, \mathrm{p}=0.024$, and $16.4 \pm$ 4.7 vs. $12.5 \pm 4.9 \mathrm{~ms}, \mathrm{p}=0.005$, respectively). In the HUT+ 
Table 4. Predictors of a positive HUT test on multivariate analysis

\begin{tabular}{llll}
\hline & $\begin{array}{l}\text { Odds } \\
\text { ratio }\end{array}$ & $\begin{array}{l}\text { 95\% confidence } \\
\text { interval }\end{array}$ & $\begin{array}{l}\mathrm{p} \\
\text { value }\end{array}$ \\
\hline FMD, \% & 1.328 & $1.081-1.630$ & 0.007 \\
Female gender & 1.725 & $0.140-2.401$ & 0.452 \\
rMSSD, ms & 1.036 & $0.951-1.129$ & 0.422 \\
pMN50, \% & 1.162 & $0.971-1.390$ & 0.101 \\
High-frequency domain, ms & 0.884 & $0.692-1.129$ & 0.322 \\
\hline
\end{tabular}

Table 5. Comparison of FMD, NMD and HRV according to the type of NMS

\begin{tabular}{lccl}
\hline & $\begin{array}{l}\text { Mixed type } \\
(\mathrm{n}=16)\end{array}$ & $\begin{array}{c}\text { Nonmixed } \\
\text { type }(\mathrm{n}=9)\end{array}$ & $\begin{array}{l}\mathrm{p} \\
\text { value }\end{array}$ \\
\hline FMD, \% & $9.0 \pm 2.1$ & $8.4 \pm 3.8$ & 0.462 \\
NMD, \% & $25.6 \pm 4.4$ & $24.6 \pm 5.2$ & 0.910 \\
Mean NN, ms & $852.4 \pm 99.9$ & $883.6 \pm 86.1$ & 0.234 \\
SDNN, ms & $148.5 \pm 34.5$ & $153.0 \pm 36.9$ & 0.932 \\
SDANN, ms & $134.2 \pm 36.5$ & $137.9 \pm 40.9$ & 0.734 \\
rMSSD, ms & $40.1 \pm 8.3$ & $36.9 \pm 12.0$ & 0.132 \\
pMN50, \% & $18.1 \pm 8.3$ & $13.8 \pm 7.3$ & 0.234 \\
High-frequency domain, ms & $17.0 \pm 4.7$ & $15.5 \pm 4.8$ & 0.295 \\
Low-frequency domain, ms & $22.0 \pm 5.2$ & $22.1 \pm 5.8$ & 0.677 \\
\hline
\end{tabular}

group, the low-frequency domain was significantly greater than that of the control group ( $22.0 \pm 5.3$ vs. $18.5 \pm 4.9$ $\mathrm{ms}, \mathrm{p}=0.019)$, whereas marginal significance was shown in comparison to the HUT-group $(22.0 \pm 5.3$ vs. $18.7 \pm$ $7.3 \mathrm{~ms}, \mathrm{p}=0.071$ ). Including FMD, sex and significantly relevant indices of $\mathrm{HRV}$, the multivariate logistic regression analysis showed that FMD was an independent predictor of positive HUT (odds ratio 1.328, 95\% confidence interval 1.081-1.630, $\mathrm{p}=0.007$; table 4 ). There were no differences in FMD, NMD and HRV between the mixed type and nonmixed type of NMS (table 5).

\section{Discussion}

In this study we demonstrated increased FMD values and elevated parasympathetic parameters on 24-hour AECG in NMS patients with a positive HUT test, which suggests that augmented endothelial function and increased parasympathetic tone are important for positive HUT test outcomes in patients with NMS. NMS refers to peripheral vasodilatation and inappropriate bradycardia leading to hypotension and loss of consciousness by parasympathetic stimulation following sympathetic activation. During NMS, atropine administration can prevent the bradycardia but not the hypotension, suggesting that marked peripheral vasodilation is one of the major causes of the fall in arterial blood pressure. It has been reported that profound vasodilation occurs in skeletal muscle and this can explain much of the hypotension associated with NMS [17]. This concept could be supported by the fact that NMS can be seen in patients who have undergone cardiac pacing, providing that prevention of bradycardia does not prevent the onset of vasovagal syncope [18].

The major site of vasodilation is the peripheral arteries in skeletal muscle. Sympathetic nerve activity is suppressed just prior to and during vasovagal attacks, indicating that sympathetic withdrawal contributes to the dilation. However, in the forearms of healthy subjects who have undergone sympathectomy or local anesthetic nerve block, the skeletal muscle vasodilation seen during syncope is greater than that caused by sympathetic withdrawal alone [19]. This observation suggests that sympathetic withdrawal alone cannot account for all of the forearm vasodilation that occurs during syncope, consistent with the possible existence of active vasodilation of peripheral arteries in skeletal muscles during NMS. One of the possible explanations is hyperactive endothelial function. Since endothelial function modulates peripheral vascular tone, increased sympathetic nerve activity during emotional or orthostatic stress increases the heart rate and cardiac output with vigorous ventricular contraction. This stimulates the mechanoreceptors of the endothelium and the production of nitric oxide (NO), and marked vasodilation of peripheral arteries could occur [20]. Although endothelial function was evaluated in a resting supine position, cuff deflation of the brachial artery, which induces temporal reactive hyperemia and increased shear stress, leads to similar conditions when the patients experience stress with sympathetic activation. Therefore, the results of the FMD will differ according to the baseline endothelial function, which responds to temporal stressors such as increased blood flow and shearing forces.

It was reported that in patients with NMS, using urinary cycle $3^{\prime}, 5^{\prime}$-guanosine monophosphate as a biological marker of NO during HUT, activity increased by $220 \%$ with NMS while in the control group it decreased by $67 \%$ [21]. Endothelial hyperactivity and upregulation of NO lead to profound vasodilation, which is much stronger than vasodilation caused by adrenergic stimulation in response to orthostatic stress alone. Studies have reported 
significantly higher FMD and higher plasma NO concentrations in people with vasovagal syndrome [22]. A previous study reported that augmented endothelial function plays an important role in the development of NMS [7]. However, the authors did not include patients with suspected NMS with negative HUT. In this study, the HUT+ group had a significantly greater FMD value than that of the HUT- group and the control group, which strongly suggests that increased endothelial function was one of the important determinants that induced positive HUT in the patients with NMS. Another possible explanation is chemoreceptor-mediated stimulation, such as acetylcholine, which activates the endothelial cells and increases the production of NO in patients with NMS [20]. Patients with NMS usually have an increased vagal activity and increased production of acetylcholine, which is a postsynaptic transmitter of the vagus nerve [23]. A previous study reported that NO mediates cholinergic neurogenic vasodilatation in the dog hind limb, evoked by activation of cholinergic nerves involving the synthesis of NO [24]. In addition, there were reports of cholinergic neurogenic vasodilation in the coronary and pulmonary arteries, in which $\mathrm{N}$-nitro-L-arginine methyl ester (an NO synthase inhibitor) specifically inhibited vasodilation in response to vagal stimulation and acetylcholine $[25,26]$. It was suggested that neuronally released acetylcholine could diffuse across the smooth muscle layer to release NO from endothelial cells. Therefore, the increased vagal tone can cause excessive vasodilation through $\mathrm{NO}$ production in patients with NMS. This could be an explanation of our results with regard to the difference in endothelial function among the study patients, of whom those in the HUT+ group had the highest FMD values. In the multivariate analysis, only FMD was an independent predictor of positive HUT. One possible explanation is that, although relevant parasympathetic indices were not significantly affected in the analysis, increased vagal activity may increase acetylcholine and NO, which could have played a role in enhanced endothelial function in this study.

Traditional measurements of HRV derived from AECG reveal the autonomic activity of the heart and provide useful information for understanding the pathophysiology of NMS $[27,28]$. Previous reports revealed that rMSSD and pNN50 reflect more autonomic control of the heart than other time-domain HRV indices, such as mean NN, SDNN and SDANN [29]. In addition, parasympathetic activation indicated by increased high-frequency spectra was noted at the onset of syncope during HUT testing [27]. In patients with NMS, it is important to clarify whether the baseline autonomic activity expressed by
HRV indices is increased or not. Our results agreed with previous reports that NMS patients with variations in vagal autonomic tone appear to be more prone to positive HUT when their parasympathetic tone is elevated [6]. In this study, the patients in the HUT- group showed significantly greater decreases than the HUT+ group in the indices of rMSSD, pNN50 and high-frequency domains, which represent parasympathetic activity. However, there was no difference in these indices between the HUTgroup and the control group. In addition, there was no difference in FMD between the HUT- group and the control group. These observations strongly suggest that even if a patient has a history of NMS, the vagal activity involved with HUT is one of the important determinants for discriminating false-negative HUT results. Therefore, if the patient has a history of NMS and low-vagal activity in HRV or decreased endothelia function, the negative HUT result is less reliable. This concept is supported by the vasoreactivity and AECG results of the control group, which were comparable with the HUT- group.

There were several potential limitations to this study. First, FMD may be influenced to varying degrees by many factors, such as cardiovascular risk factors and vasoactive medications. In this study we excluded patients with hypertension and diabetes. The current smokers had refrained from smoking $12 \mathrm{~h}$ before the study. In addition, there were no differences among the groups in the levels of smoking and dyslipidemia. Second, for patients in the HUT- group, the HUT test was not repeated and we did not perfectly exclude other causes of syncope besides NMS. However, the patients were young subjects with typical symptoms of NMS and all of their comprehensive medical examinations were negative, including the cardiovascular and neurological studies. Third, the total number of patients was relatively small. Further studies including a greater number of patients will clarify the associations among endothelial function, parasympathetic autonomic activity and the results of HUT testing in patients with NMS.

\section{Conclusions}

In patients with NMS, not only baseline parasympathetic activity but also endothelial function may affect the results of the HUT test.

\section{Conflict of Interest}

We have no commercial associations that might pose a conflict of interest in connection with the presented work. 


\section{References}

1 Mosqueda-Garcia R, Furlan R, Tank J, Fernandez-Violante $\mathrm{R}$ : The elusive pathophysiology of neurally mediated syncope. Circulation 2000;102:2898-2906.

2 Benditt DG: Neurally mediated syncopal syndromes: pathophysiological concepts and clinical evaluation. Pacing Clin Electrophysiol 1997;20:572-584.

3 Grubb BP, Wolfe D, Temesy-Armos P, Hahn $\mathrm{H}$, Elliott L: Reproducibility of head upright tilt table test results in patients with syncope. Pacing Clin Electrophysiol 1992;15:14771481.

4 Brooks R, Ruskin JN, Powell AC, Newell J, Garan H, McGovern BA: Prospective evaluation of day-to-day reproducibility of upright tilt-table testing in unexplained syncope. Am J Cardiol 1993;71:1289-1292.

5 Hosaka H, Takase B, Katsushika S, Ohsuzu F, Kurita A: Altered fractal behavior and heart rate variability in daily life in neurally mediated syncope. Biomed Pharmacother 2003; 57(suppl 1):77s-82s.

6 Kochiadakis GE, Kanoupakis EM, Rombola $\mathrm{AT}$, Igoumenidis NE, Chlouverakis GI, Vardas PE: Reproducibility of tilt table testing in patients with vasovagal syncope and its relation to variations in autonomic nervous system activity. Pacing Clin Electrophysiol 1998 21:1069-1076.

7 Galetta F, Franzoni F, Plantinga Y, Ghiadoni L, Merico G, Tocchini L, Braccini L, Rossi M, Carpi A, Taddei S, Santoro G: Endothelial function in young subjects with vaso-vagal syncope. Biomed Pharmacother 2006;60: 448-452.

8 Santini L, Capria A, Brusca V, Violo A, Smurra F, Scarfo I, Forleo GB, Papavasileiou LP, Borzi M, Romeo F: An increased endothelialindependent vasodilation is the hallmark of the neurally mediated syncope. Clin Cardiol 2012;35:107-110.
9 Task Force of the European Society of Cardiology and the North American Society of Pacing and Electrophysiology: Heart rate variability: standards of measurement, physiological interpretation and clinical use. Circulation 1996;93:1043-1065.

10 Raviele A, Giada F, Brignole M, Menozzi C, Marangoni E, Manzillo GF, Alboni P: Comparison of diagnostic accuracy of sublingual nitroglycerin test and low-dose isoproterenol test in patients with unexplained syncope. Am J Cardiol 2000;85:1194-1198.

11 Morillo CA, Klein GJ, Zandri S, Yee R: Diagnostic accuracy of a low-dose isoproterenol head-up tilt protocol. Am Heart J 1995;129: 901-906.

12 Carlioz R, Graux P, Haye J, Letourneau T, Guyomar Y, Hubert E, Bodart JC, Lequeuche B, Burlaton JP: Prospective evaluation of high-dose or low-dose isoproterenol upright tilt protocol for unexplained syncope in young adults. Am Heart J 1997;133:346-352.

13 Park J, Jang SY, Yim HR, On YK, Huh J, Shin DH, Kim JH, Kim JS: Gender difference in patients with recurrent neurally mediated syncope. Yonsei Med J 2010;51:499-503.

14 Huang A, Sun D, Koller A, Kaley G: Gender difference in flow-induced dilation and regulation of shear stress: role of estrogen and nitric oxide. Am J Physiol 1998;275:15711577

15 Arora S, Veves A, Caballaro AE, Smakowski P, LoGerfo FW: Estrogen improves endothelial function. J Vasc Surg 1998;27:1141-1146.

16 Brignole M, Menozzi C, Del Rosso A, Costa S, Gaggioli G, Bottoni N, Bartoli P, Sutton R: New classification of haemodynamics of vasovagal syncope: beyond the VASIS classification: analysis of the pre-syncopal phase of the tilt test without and with nitroglycerin challenge - Vasovagal Syncope International Study. Europace 2000;2:66-76.

17 Dietz NM, Joyner MJ, Shepherd JT: Vasovagal syncope and skeletal muscle vasodilatation: the continuing conundrum. Pacing Clin Electrophysiol 1997;20:775-780.
18 el-Bedawi KM, Wahbha MA, Hainsworth R Cardiac pacing does not improve orthostatic tolerance in patients with vasovagal syncope. Clin Auton Res 1994;4:233-237.

19 Barcroft H, Edholm OG: On the vasodilatation in human skeletal muscle during posthaemorrhagic fainting. J Physiol 1945;104: 161-175.

20 Adams MR: Clinical assessment of endothelial function. Endothelium 2006;13:367-374.

21 Kaufmann H: Neurally mediated syncope: pathogenesis, diagnosis, and treatment. Neurology 1995;45:S12-S18.

22 Pietrucha AZ: Endothelial function in vasovagal syncope. Expert Rev Cardiovasc Ther 2014;12:1387-1389.

23 Takase B, Akima T, Uehata A, Katushika S, Isojima K, Satomura K, Ohsuzu F, Kurita A: Endothelial function and peripheral vasomotion in the brachial artery in neurally mediated syncope. Clin Cardiol 2000;23:820-824.

24 Loke KE, Sobey CG, Dusting GJ, Woodman OL: Cholinergic neurogenic vasodilatation is mediated by nitric oxide in the dog hindlimb. Cardiovasc Res 1994;28:542-547.

25 Broten TP, Miyashiro JK, Moncada S, Feigl EO: Role of endothelium-derived relaxing factor in parasympathetic coronary vasodilation. Am J Physiol 1992;262:H1579-H1584.

26 McMahon TJ, Hood JS, Kadowitz PJ: Pulmonary vasodilator response to vagal stimulation is blocked by $\mathrm{N}^{\omega}$-nitro-L-arginine methyl ester in the cat. Circ Res 1992;70:364-369.

27 Lipsitz LA, Mietus J, Moody GB, Goldberger AL: Spectral characteristics of heart rate variability before and during postural tilt: relations to aging and risk of syncope. Circulation 1990;81:1803-1810.

28 Vybiral T, Bryg RJ, Maddens ME, Boden WE: Effect of passive tilt on sympathetic and parasympathetic components of heart rate variability in normal subjects. Am J Cardiol 1989; 63:1117-1120.

29 Stein PK, Kleiger RE: Insights from the study of heart rate variability. Annu Rev Med 1999; 50:249-261.
Endothelial and Autonomic Factors in Vasovagal Syncope 